\title{
Analysis of Delayed Dislocation of IoL: Cause And Effect
}

\author{
1,*'Dr. Sanjoy Chowdhury, M.S., Dnb, D. O., \\ ${ }^{2}$ Dr.Priyanka ${ }^{2}$ Dr. Snehakumari, ${ }^{3}$ Drnilanjanchowdhury \\ ${ }^{1}$ Joint Director (Medical And Health Services), Head Of Ophthalmology, Bokaro Generalhospital, \\ Steel Authority Of India Limited \\ ${ }^{2}$ DNB Resident, ${ }^{3}$ Intern,Bokaro General Hospital, Steel Authority Of India Limited
}

\begin{abstract}
Aim: To analyse risk factors associated with delayed dislocation of IOLs.

Methods: Hospital based prospective intervention study during 1997-2012.All cases of IOLs dislocated after 6 months were included. Investigations included B scan and MRI was done in cases with h/o trauma. Multivariate analysis to detect risk factors was done

Results: Total 25 cases of delayed dislocation out of 12000 pseudophakic. Mean age: 63.2 years, with M: F-5:1. Mean interval: 6.5 years. 12 Iris claw lenses dislocated in $A C$, easily fixated with good visual outcome, 3 cases of PCIOL with IOFB detected on MRI,5 cases of trauma with perfect capsulorrhexis, 2 cases of pseudo exfoliation , 2 cases had longer axial length $(>26 \mathrm{~mm})$.All cases had significant visual loss.

Multivariate analysis confirmed occult IOFB, trauma, iris fixation, long axial length as risk factors.

Conclusion: Undetected IOFB, iris atrophy and larger eyeball may predispose to delayed dislocation of IOL
\end{abstract}

\section{Introduction}

Dislocation of IOL is one of the serious complication after cataract surgery. It's a delayed complication that is presented after months or years (minimum 6 months) which can take cataract surgeons by surprisespontaneous IOL dislocation years after the original surgery. For many ophthalmologists, the problem comes out of the blue, as the surgery had gone uneventful.

Iris claw lens could particularly be at risk on long-term because of atrophic changes. This study has become relevant due to resurgence of new generation iris claw lenses as a part of refractive surgical procedure particularly in high myopic. Long axial length and atrophic changes may lead to delayed dislocation. The patient will experience blurring of vision as they are not looking through the center of the IOL anymore while some may actually see the edge of the dislocated IOL.There can be diplopia as well. Patients naturally get very anxious and it may lead even the surgeon to press the 'panic button'.Zonular dialysis is one of the thought that comes to the mind and the surgeon starts to find the reason behind it to be presented so late.Detailed history of patient after the surgery is taken,looking for any possible reasons like trauma. Trauma is one of the most common cause behind IOL dislocation and when patient recalls, it is easier to take that as a major factor for this presentation.

But the scenario might not be straight forward every time!One such condition is occult intraocular foreign body.It could have gone into patient's eye with minimal entry port and not much of a sign of damage of external serous coats especially conjunctiva.Here, patient presents with less inflammation than expected. Small sharp objects can get inside eye with great velocity in workers of metal industry. So there can be relief after inflammation subsides and the incidence is forgotten thereafter,so much that it might not be mentioned when asked about the history of trauma. It is obvious to expect men to be victim of intraocular foreign body more than women,as most of the patients are the workers and the labourers.

Meanwhile the foreign body is settled inside the eye and chronic inflammation \& subsequent fibrosis sets in.The location of the foreign body will decide the further course of its complications.If the foreign body is located anywhere near the IOL,the fibrosis will cause tractional force \& lead to dislocation of IOL.

\section{Materials \& Methods}

This is a prospective study performed at the department of ophthalmology, Bokaro General Hospital, Bokaro Steel City,Jharkhand. All the patients presenting with delayed dislocation of IOL presenting during 1997 -2012 were included in the study.

\section{Exclusion Criteria:}

1. Patients presenting under 6 month of surgery.

2. Intraoperative complications of any kind i.e. Raised IOP,Vitreous loss.

3. Postoperative complications of any kind i.e.Hyphema, TASS 


\section{H/o surgical or laser intraocular intervention i.e.PCR}

Detailed history of ocular as well as systemic diseases were taken. Visual acuity was noted with full correction. Slit lamp examination was performed.IOP was measured by applanation tonometer. Fundus examination was done by indirect ophthalmoscopy and slit lamp biomicroscopy with 90 D.Every patient then underwent B scan ocular ultrasonography.

Routine investigations of blood and X-ray chest was done.

It was followed by MRI (orbit) in suspected retained IOFB cases.

\section{Results}

Mean age of the patients was 63.2 years.

Male: female ratio was 4.5:1. (18 M: $04 \mathrm{~F})$

Mean interval of presentation after surgery was found to be 6.5 years.

12 of the total patients were implanted Iris Claw Lens. i.e. 12/22 (54.5\%)

5 of the total patients had history of trauma. i.e. $5 / 22$ (22.72)

3 of the total patients had occult intraocular foreign body.i.e. $3 / 22(13.63)$

2 of the total patients had pseudo exfoliation syndrome i.e. $2 / 22(9 \%)$

Table 1 Distribution Of Causes Of IOL Dislocation According To Sex:

\begin{tabular}{|c|c|c|c|c|}
\hline Sex & Iris Claw Lens & Trauma & Intraocular Fb & Pseudoexfoliation \\
\hline Male & 10 & 05 & 03 & - \\
\hline Female & 02 & - & - & 02 \\
\hline
\end{tabular}

Table 2 Chi-Square Calculator

The contingency table below provides the following information: the observed cell totals, (the expected cell totals) and [the chi-square statistic for each cell].

\begin{tabular}{|c|c|c|c|c|c|}
\hline \multicolumn{6}{|l|}{ Results } \\
\hline & ICL & TRM & IFB & PEX & Row Totals \\
\hline $\mathbf{M}$ & $10(9.82)[0.0]$ & 5 (4.09) [0.20] & $3(2.45)[0.12]$ & $0(1.64)$ [1.64] & 18 \\
\hline $\mathbf{F}$ & $2(2.18)[0.02]$ & $0(0.91)[0.91]$ & $\begin{array}{lll}0 & (0.55) & {[0.55]}\end{array}$ & $2(0.36)[7.36]$ & 4 \\
\hline $\begin{array}{l}\text { Column } \\
\text { Totals }\end{array}$ & 12 & 5 & 3 & 2 & $\begin{array}{l}22 \text { (Grand } \\
\text { Total) }\end{array}$ \\
\hline
\end{tabular}

The chi-square statistic is 10.7963 . The P-Value is 0.01288 . The result is significant at $\mathrm{p}<0.05$.

Table value of chi-square with degrees of freedom (2-1) (4-1) =3 at 0.05 level of significance $=7.81$.

Since the calculated value of chi-square is greater than the table value, the hypothesis that there is no association between sex and the causes is rejected.

Therefore, we conclude that there is a strong association between sex and the causes.

\section{Discussion}

When a case of dislocation of IOL comes to the ophthalmologist months or years after the surgery,history taking becomes one of the key step to come to the diagnosis. In our study, we tried to find out the possible causes for delayed dislocation of IOL. More than half of the patients (i.e.54.5\%) had Iris Claw -IOL implanted suggesting that the type of implanted lens has got significant influence this outcome as, no other cause was identified in these patients. Both the patients in which axial length was found to be $>26 \mathrm{~mm}$, had Iris Claw - IOL implanted in them. All the patients with history of trauma and occult intraocular FB were male workers \& labourers. These patients are always at risk as a part of professional hazards and thorough imaging should be integral part in work up of these cases.

In the statistical analysis, we found strong association between sex of the patient and the cause behind the dislocated IOL. It was due to the fact that male workers are naturally exposed to trauma at work or entry of foreign body inside the eye through penetrating wound. Thus here was gender predilection for these causes. If the treating ophthalmologist keep this in mind, it would be easier to reach to possible causes. Also it throws light on the need of proper protective measures for the industrial workers at their place of work.

\section{Conclusion}

Iris claw lens is the commonest type to be dislocated, so one should always think twice before implanting as refractive procedure. Occult IOFB and trauma can cause to delayed dislocation of IOL. 


\section{References}

1. S L Pueringer, D O Hodge, J C Erie. Risk of Late Intraocular Lens Dislocation after Cataract Surgery, 1980-2009: A Population-Based Study. Am J Ophthalmol 2011;152:618-623

2. Shingleton BJ, Yang Y, O’Donoghue MW. Management and outcomes of intraocular lens dislocation in patients with pseudo exfoliation. J Cataract Refract Surg 2013; 39: 984-93.

3. Hayashi K, Hirata A, Hayashi H. Possible predisposing factors for in-the-bag and out-of-the bag intraocular lens dislocation and outcomes of intraocular lens exchange surgery. Ophthalmology 2007; 114:969-75.

4. Monestram EI. Incidence of dislocation of intraocular lenses and pseudophakodonesis 10 years after cataract surgery. Ophthalmology 2009; 116:2315-320.

Fig 1 Dislocated ICIOL (Nasal clip)
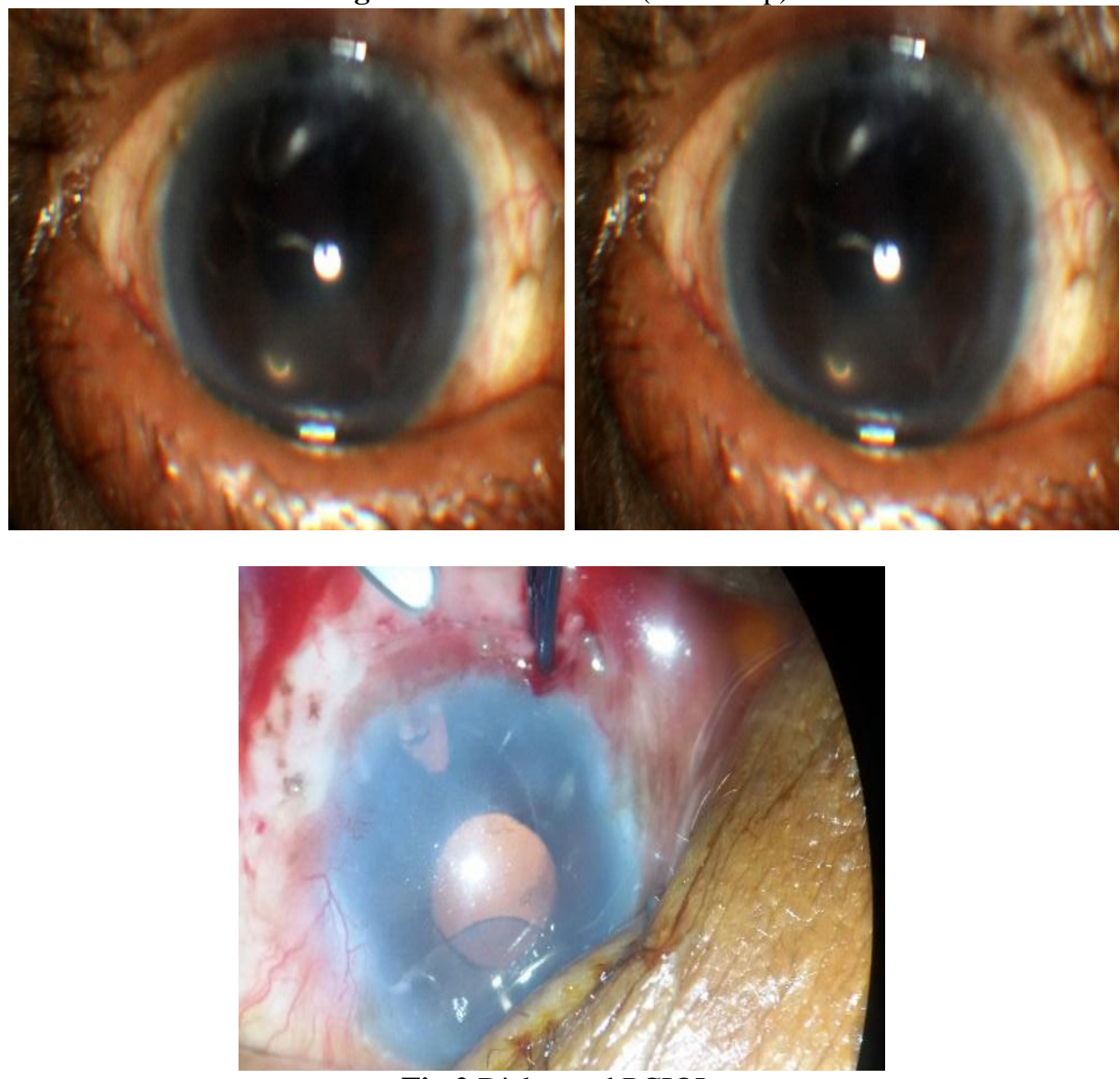

Fig 2 Dislocated PCIOL

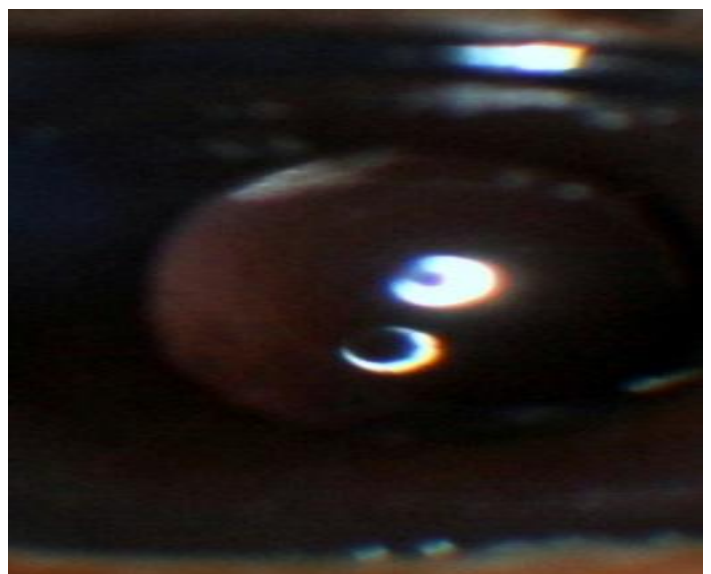

Fig 3 Steps of refixation of ICIOL 

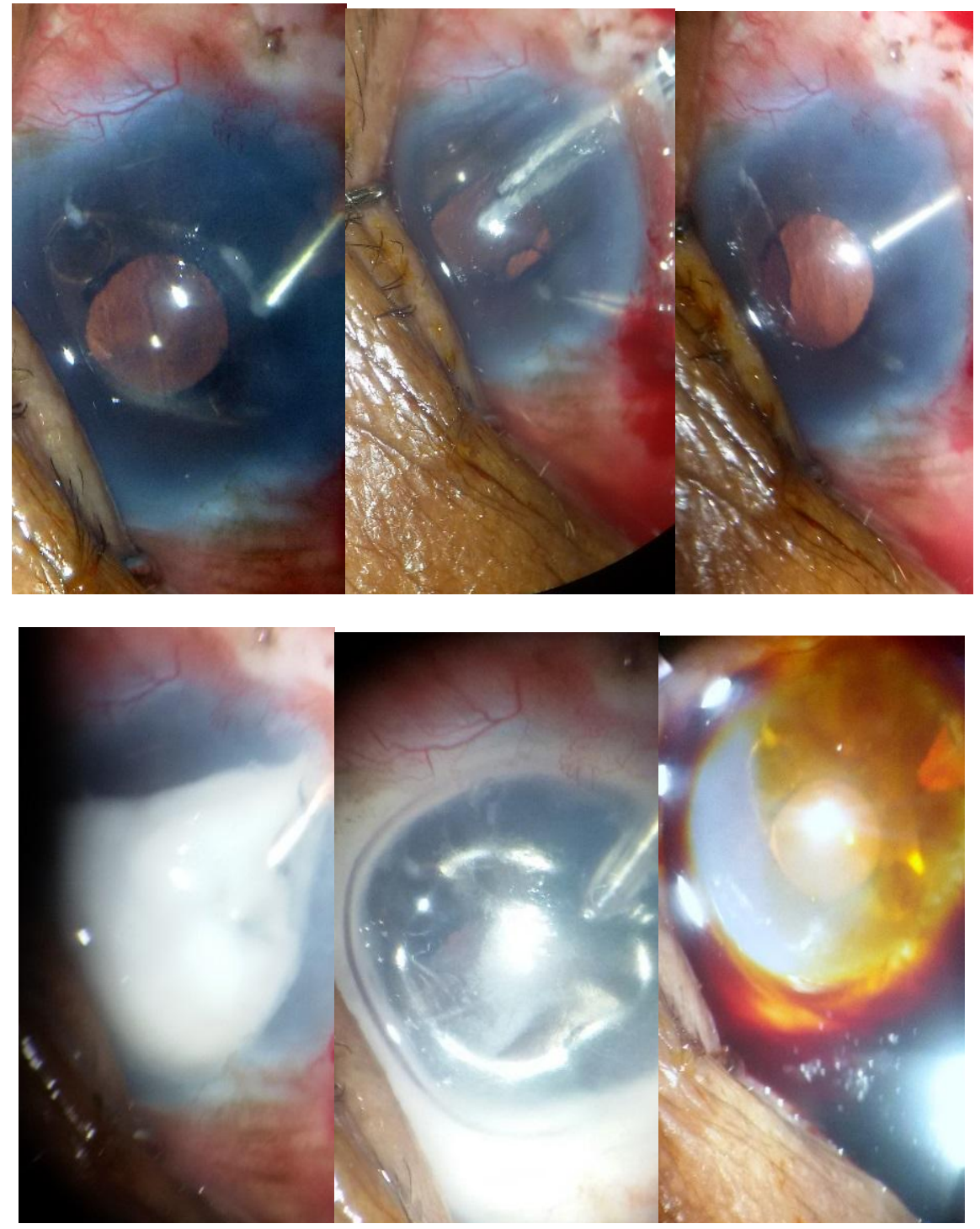

Fig 4 Re fixated ICIOL

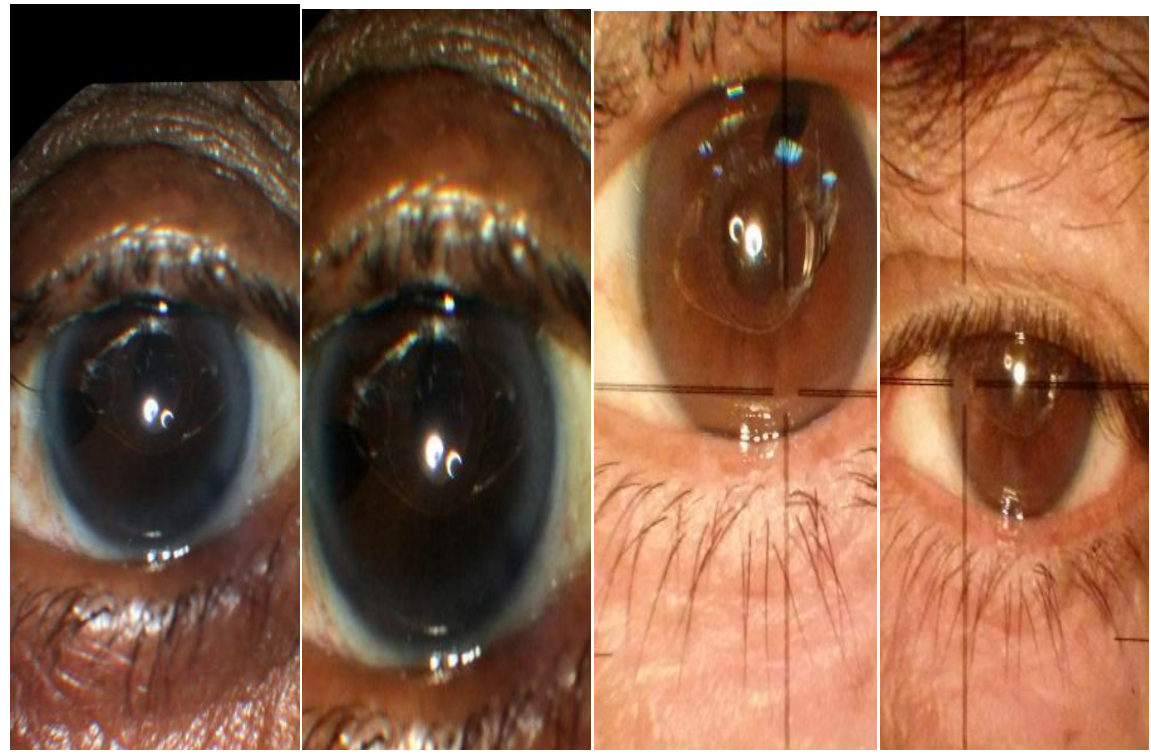




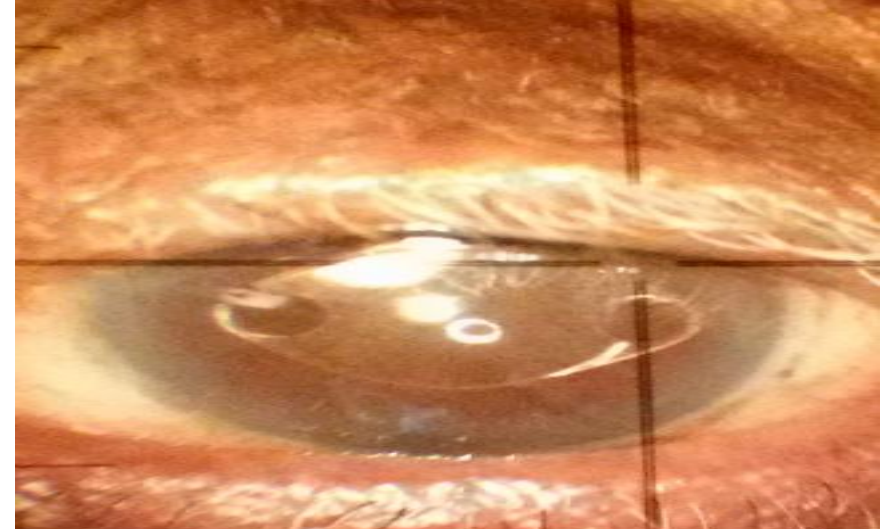

Fig 5 Removed ICIOL : widening of iris clip

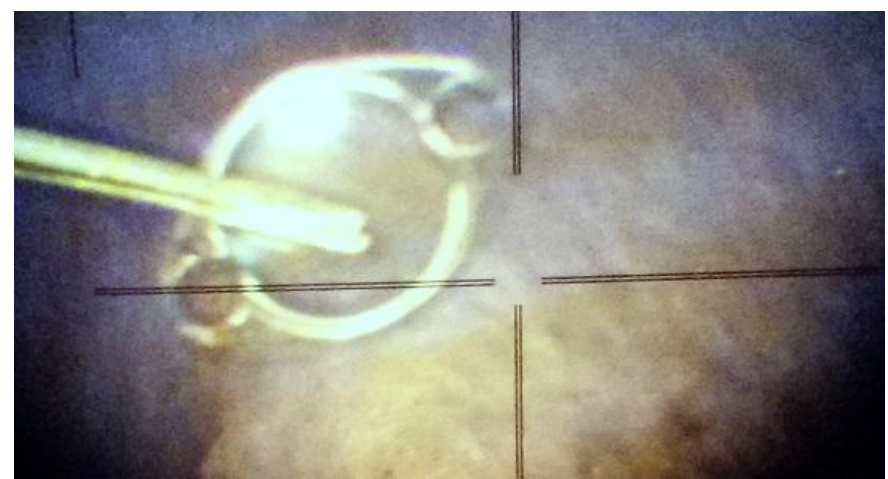

Fig 6 Retained IOFB causing dislocation of IOL after 8 years

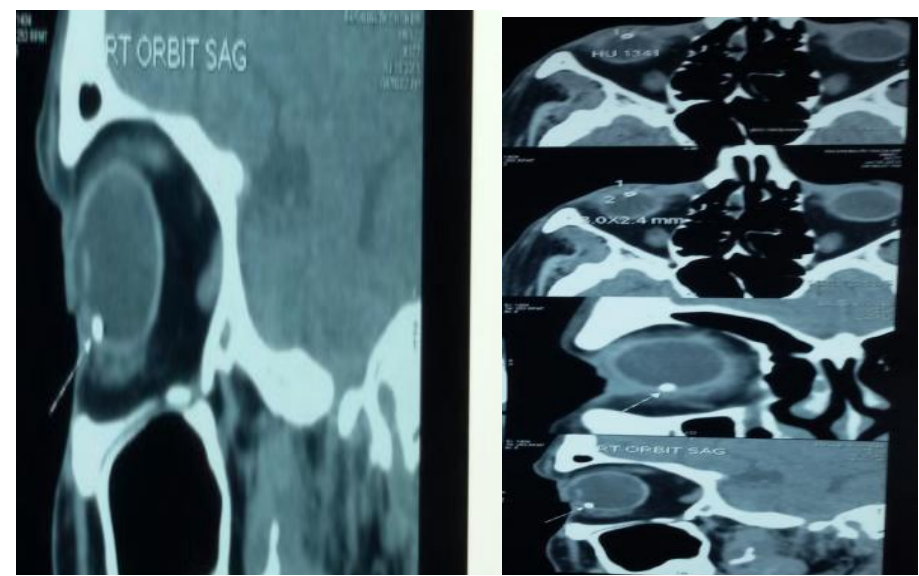

Fig 7 Graphical representation of distribution of patients according to the causes:

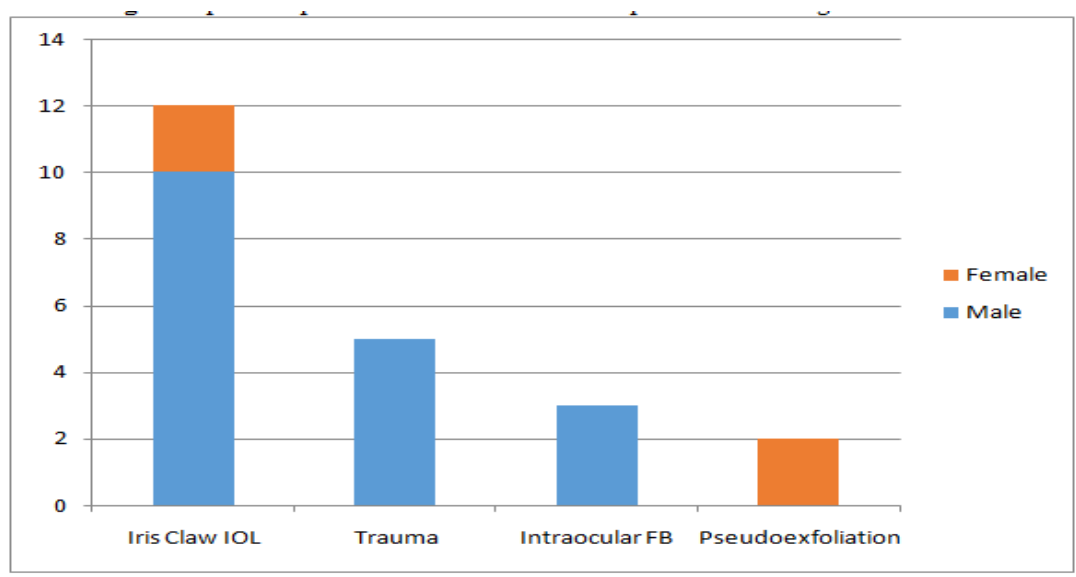

